\title{
ENERGY BALANCED AND EFFICIENT CLUSTERING METHOD FOR WIRELESS SENSOR NETWORKS
}

\author{
Yong Chan Lee ${ }^{1}$, Yong Hak Sin ${ }^{2}$, Won Chol Jang ${ }^{3}$ and Un Kyong Choe ${ }^{4}$ \\ ${ }^{1,3}$ College of Information Science, Kim Il Sung University, Democratic People's Republic of Korea \\ ${ }^{2,4}$ Institute of Information Science, Kim Il Sung University, Democratic People's Republic of Korea
}

\begin{abstract}
In this paper the energy balanced and efficient clustering method based on balance of energy consumption of nodes in WSN is proposed, which may be applied to any WSN. The almost static centralized protocol that differs from previous methods is proposed, the main feature of which is that the sinks transmit most of control message and process most of data. First, EBEC method is proposed, which optimizes by considering energy consumption on transmitting and receiving data, energy consumption on the reclustering and hot-spot problem that be optimized individually in previous works. In order to implement this method, VW BAK-C algorithm is used by introducing the concept of variable weighted Euclid distance to $k$-clustering algorithm. Second, the previous clustering methods are classified into random method and the method based on QoS according to the characteristic of cluster head rotation, and average of total energy consumption of nodes is analyzed mathematically. The proposed method is compared and analyzed. Third, the performance of the proposed method is evaluated by comparing with other clustering methods through simulation.
\end{abstract}

Keywords:

WSN, EBEC, Clustering, QoS, Energy Consumption

\section{INTRODUCTION}

In general, wireless sensor network consists of a large number of sensor nodes and a sink deployed over sensing area [1]. Every sensor node gathers data and sends data to sink by multi-hop communication and the sink is connected to master computer. Usually gathered data is sent from nodes to sink by clustering, since it is not efficient in terms of energy for every node to send the gathered data directly to sink.

MAC protocols in WSNs can be divided into two main categories: schedule-based protocols and contention-based protocols. Schedule-based protocols use clustering. The intensive research on schedule-based MAC protocols has been done due to the characteristics of WSN. The most representative protocol is LEACH (Low-Energy Adaptive Clustering Hierarchy), on which most clustering methods are based. The disadvantage of LEACH is variance of the number of cluster heads, the existence of FCH (Forced Cluster Head), the non-uniformity of cluster size and low bandwidth efficiency due to using CDMA to achieve communication between clusters and so on.

Since the regional distribution of cluster head nodes is random and two cluster heads may be distributed nearby, there may be a cluster head with no element. This cluster head is called FCH and it clarified that in case that optimal cluster head probability is $8 \%$ among 1000 nodes $\mathrm{FCH}$ is $1.23 \%$, which shows that $15 \%$ of cluster heads are FCHs [23]. The other cluster heads are called $\mathrm{VCH}$ (Volunteer Cluster Head).
The random distribution of cluster heads makes cluster size very unbalanced, which accompanies energy consumption. CDMA communication between clusters is used to reduce interference between clusters, but it results in deteriorating the overall performance of the network by low bandwidth efficiency. In addition it requires for all nodes to have hardware for CDMA communication. In order to overcome these disadvantages, several clustering methods have been proposed [2 31].

Clusters can be classified in several ways: homogeneous and heterogeneous clusters or static and dynamic clusters.

Some networks classifies nodes into RFD (Reduced Function Device) and FFD (Full Function Device) and nodes with high performance can be cluster heads. On the other hand, since nodes with higher performance usually become static cluster heads in heterogeneous networks, static clustering is easy.

However, WSN usually uses homogeneous and dynamic clustering. Static clustering is easy to deploy, but is only appropriate for limited scenarios where the sensor field is predetermined, the targets to monitor are not in motion and maintenance is easy (i.e. sensors replacements) in the network [12]. Dynamic clustering gives latency and energy consumption in forming cluster, but can be used for both heterogeneous and homogeneous cluster and consumes less energy on transmitting sensing data. Also reforming clusters gives easy tracking of moving targets.

Communication between nodes in WSN based on clustering consists of communication in clusters (communication between nodes and cluster heads) and communication between clusters (communication between cluster heads, or cluster heads and sink, in some cases of communication between cluster heads and nodes).

Clustering protocols can be divided into protocols based on LEACH and reactive protocols. In LEACH-C (LEACHCentralized), the nodes selected as cluster heads have to report to the sink that they are cluster heads to avoid unbalanced concentration of cluster heads.

After the sink that is aware of positions of nodes receives messages from the nodes selected as cluster heads, it requires for some of the cluster heads not to be cluster heads to reduce the number of FCHs.

The characteristic in PEGASIS (Power-Efficient Gathering in Sensor Information System) is that it forms a chain connecting near nodes and alternates cluster heads according to the chain. One of the assumptions in protocol based on LEACH is that nodes always have data to send.

There are HEED, EECS, SA, ACE and TASC in these protocols. In HEED, it elects new cluster head taking into account three factors: neighbor node of previous cluster heads, node with 
highest degree and node with highest residual energy. However, it results in unbalanced distribution of cluster heads.

ACE aimed at uniform distribution of nodes in clusters, which removes FCH. TASC (Topology Adaptive Spatial Clustering) supports partial uniformity of distribution of cluster heads. It avoids duplicate inclusion of a node. TEEN, APTEEN, CAG, and $\mathrm{U}-\mathrm{CAG}$ are reactive protocols of WSN, in which clustering is triggered by particular event. TEEN (Threshold-sensitive Energy Efficient sensor Network Protocol) is a clustering protocol which reacts immediately on the rapid change of sensing data. Each node takes turns of cluster head with the period of $\mathrm{T}$ that is called as cluster period (cluster forming is very similar in LEACH). In APTEEN (Adaptive Periodic Threshold-sensitive Energy Efficient sensor Network Protocol) selection process of cluster head is same in LEACH-C. Wait time for data report of nodes are reduced using fixed threshold, soft threshold, and timer. CAG (Clustered Aggregation technique) is an aggregation algorithm to collect data using correlation between sensing data. In U-CAG a cluster consists of the nodes that obtain similar sensing value and is not reformed unless the sensing data is in certain limitation.

Clustering protocols may be divided into $\mathrm{FH}$ (first-cluster Head) and FC (First-Cluster) scheme, based on the order of clustering and selection of cluster head. Most protocols are $\mathrm{FH}$ scheme. In [2], FC clustering method is proposed, which divides sensor nodes into different clusters in the way of recursive bisection using PSO (Particle Swarm Optimization) and elects cluster heads. Of course this clustering method is optimal, but the cost of cluster forming is high since it is dynamic clustering and the computation of PSO algorithm is NP-hard.

In [3], it clarified the hierarchical cluster that it considers the cluster as one macro node and proposed semi-dynamic clustering method. It models clusters in graph structure and the graph is not changed unless the weight value of sides - communication cost are not changed in a certain limit.

In MINA (Multi-hop Infrastructure Network Architecture), TDMA is employed inter-cluster communication and FDMA is employed among clusters [6].

Centralized clustering algorithm, SPY algorithm [7] allowed to reduce by $63 \%$ the number of necessary messages to build cluster compared to natural method and by a factor of 10 the cost of cluster formation with respect to LEACH-C method.

However, this algorithm can be used in only case that the sensor nodes are FFD, not RFD, that is, it can control transmit energy of nodes automatically.

In [8], it considered a cluster head selection approach using Analytical Hierarchy Process (AHP) and referred that it selects a cluster head by constructing the evaluation matrix based on residual energy of nodes, mobility of nodes and the distance to the involved cluster centroid.

In [9], it proposed RDCM (Randomly Delayed Clustering Method) and compared it with LEACH and ideal CNS in terms of communication energy costs.

CNS is a concept that the target or the source to sense is on the centre of the cluster and it was implemented by CBERRP (Clustering-Based Expanding-Ring Routing Protocol) in [30].

In [10], it proposed QAC (QoS-based Adaptive Clustering algorithm) where the cluster head is on the centre of the cluster but it becomes the node of other cluster similarly in [17]. In [11], it proposed the distributed clustering algorithm, LNCA (Local Negotiated Clustering Algorithm) in data gathering WSN.

The main characteristic is that it reduces energy of data transmission than in LEACH by aggregating data, but energy of cluster forming is larger than in LEACH. TCF (Two-phase Cluster Formation algorithm) formed cluster by electing cluster head according to residual energy and QoS of nodes [13] and LCA (Linked Clustering Algorithm) formed cluster by electing cluster head according to ID of nodes [14].

Besides, several clustering protocols such as distributed clustering algorithm, BSCA (BS Clustering Algorithm) [14]that elects randomly cluster heads similarly in LEACH-C, CBERRP(Clustering-Based Expanding-Ring Routing Protocol) [15], FLOG, MOCA (Multi-hop Overlapping Clusters Algorithm) [17], LENO (Least Rotation Near-Optimal) [18], WCA (Weighted Clustering Algorithm), DAC (Distributed Asynchronization Clustering) [19], MICA (MICa Adjustments) [20], EESH (Energy Efficient Strong Head clustering algorithm) [20] have been presented.

By analyzing several clustering methods, many researches on DDR (Distributed Dynamic Random) about homogeneous networks usually has been done. In distributed clustering the number of control messages can be reduced much by clustering by nodes, but energy consumption due to exchange of messages between nodes themselves is high. In centralized clustering, energy consumption in nodes is low, but it is not scalable. That is, when a node is added to cluster or it destroys and it is damaged, it is difficult to reconstruct about it.

When the topology of network changes, dynamic clustering is more adaptable to it compared to static clustering, but its energy consumption necessary to reconstruct cluster is high. In general, it is very steady, but it causes hot-spot problem and it is difficult to implement CNS in static clustering.

In order to solve the hot-spot problem, it rotates cluster heads in static clustering too, but it consumes additional energy because it cannot implement $\mathrm{CNS}$ in this case. Many researches on election of cluster heads based on QoS using residual energy, degrees of nodes and the distance from node to target have been done.

By using this method, energy consumption is balanced compared with random election of cluster heads, but it consumes energy because nodes have to report QoS values with data reporting. Moreover, if above processing is done in distributed clustering, nodes must have more intelligent functions. On the other hand, random election of cluster heads has disadvantages such as unbalanced distribution of cluster heads and unbalanced size of cluster.

As the election method of cluster heads [24] is based on scheduling, it consumes no energy necessary to elect cluster heads, but it is not scalable and it causes balance problem of energy consumption due to static clustering. Also, since first election of cluster heads is random, unbalanced problem in early cluster forming adheres during network lifetime.

Clustering in WSN aims at reducing energy consumption of nodes. Distributed clustering that has been addressed in most of researches is scalable, but it is less steady than centralized clustering. Also random method and QoS based method of 
election cluster heads consumes energy and has some latency delay and require hardware respectively.

In dynamic clustering it consumes much energy necessary to cluster forming and in static clustering it consumes much energy necessary to transmitting data.

In almost works except [4], [5], [16], they aims at minimizing energy necessary to transmitting and receiving data and have not analyzed energy as the sum of energy necessary to cluster forming, transmitting and receiving data after cluster forming and gathering data and calculation.

In this paper, first we propose semi-dynamic and centralized clustering method minimizing energy necessary to cluster forming. The early clustering of the proposed method becomes a combination of FLOG, BAK-C and EEUC.

Second, we evaluate the performance such as total energy consumption, balance of energy consumption and the balance of the number of nodes per cluster by modelling the proposed clustering method mathematically.

\section{PROPOSED \\ METHOD \\ (ENERGY EFFICIENT AND ENERGY CLUSTERING IN WSN) \\ BALANCE}

Clustering method proposed in this paper is FC clustering method and consists of three stages: cluster set up and election of cluster heads and rotation, data gathering and transmitting and receiving and cluster adjustment for energy balance.

\subsection{SEMI-DYNAMIC ENERGY BALANCE CLUSTERING}

\section{Assumption:}

- The sinks of WSN are aware of positions of all nodes.

- Almost no nodes are mobile and all nodes have uniform and limited energy in WSN.

- The sinks are powered, so energy consumption in the sink is not considered in WSN

- WSN is data gathering or event detective one. Although event detective WSN, it detects events of some frequency not very rare events such as fire detecting.

\subsubsection{Cluster Setup Stage:}

When there are $N$ nodes in $2 a \times 2 a$ sensing area, if it divides into clusters of which have same $P_{o p t} \cdot N$ size areas, the number of every cluster forms a sample of a Poisson distribution where $\lambda_{1}=P_{o p t}^{-1}$. Energy consumption on once data gathering in a cluster is as following,

$$
\begin{gathered}
E_{\text {cluster }}=E_{C H}+(n-1) E_{\text {non-CH }} \\
E_{C H}=(n-1) l E_{D A}+l E_{\text {elec }}+l \varepsilon_{1} d_{C H-C H}^{4}+(n-1) l E_{\text {cal }} \\
E_{\text {non-CH }}=l E_{D A}+l E_{\text {elec }}+l \varepsilon_{2} d_{\text {node-CH }}^{2}
\end{gathered}
$$

where, $E_{C H}$ is energy that cluster heads consumes, $E_{n o n-C H}$ is energy that sensor nodes consume, $l$ is the bit length of data, $E_{D A}$ is energy necessary to receiving one bit of data, $E_{\text {elec }}$ is energy necessary to starting up to transmit one bit of data, $E_{c a l}$ is energy necessary to calculate one bit of data, $\varepsilon_{1}$ and $\varepsilon_{2}$ energy necessary to propagating one bit respectively, $d_{C H-C H}$ is the communication distance between clusters, $d_{\text {node- } \mathrm{CH}}$ is the communication distance between sensor nodes and cluster heads.

Above equations are the results using CCM (Cluster Consumption Model) in [20] and is based on assuming that the number of data bits transmitted by cluster heads is same as the number of data bits from sensor nodes by taking into account data gathering, that is, the ratio of data gathering is $(n-1)^{-1}$. Because we can consider that cluster heads transmit $l$ bits of data by data gathering, if sensor nodes in every cluster gather $l$ bits of data, respectively.

Without loss of generality, $l=1$ in above equations.

$$
\begin{aligned}
E_{\text {cluster }} & =2(n-1) E_{D A}+n E_{\text {elec }}+(n-1) E_{\text {cal }} \\
& +\varepsilon_{1} d_{C H-C H}^{4}+\varepsilon_{2}(n-1) d_{\text {node- } C H}^{2}
\end{aligned}
$$

where, $n$ is the number of sensor nodes in one cluster and random variable following Poisson distribution where $\lambda_{1}=P_{o p t}^{-1}$.

Energy consumption in total network is as following,

$$
\begin{aligned}
E_{n e t} & =\sum_{i=1}^{N \cdot P_{\text {opt }}} E_{\text {cluster }}^{(i)} \\
& =\sum_{i=1}^{N \cdot P_{\text {opt }}}\left\{\begin{array}{l}
{\left[2\left(n_{i}-1\right) E_{D A}+n_{i} E_{\text {elec }}+\left(n_{i}-1\right) E_{\text {cal }}+\right.} \\
\left.\varepsilon_{1} d_{C H-C H}^{4}(i)+\varepsilon_{2}\left(n_{i}-1\right) d_{\text {node-CH }}^{2}(i)\right]+E_{\text {relay }}^{(i)}
\end{array}\right\}
\end{aligned}
$$

where, $E_{\text {relay }}^{(i)}$ is the energy consumption when $i^{\text {th }}$ cluster relays data from other cluster heads

In this paper, we considered only case that cluster heads do not gather and process data of other cluster heads and relay. When $N \cdot P_{\text {opt }}$ is large ( $N$ is usually larger than $1000, P_{\text {opt }}=8 \%$ ), energy consumption in total network is as following,

$$
E_{n e t}=N \cdot P_{o p t} E\left[\begin{array}{l}
2\left(n_{i}-1\right) E_{D A}+n_{i} E_{\text {elec }} \\
+\left(n_{i}-1\right) E_{\text {cal }}+\varepsilon_{1} d_{C H-C H}^{4} \\
+\varepsilon_{2}\left(n_{i}-1\right) d_{\text {node-CH }}^{2}(i)
\end{array}\right]+\sum_{i} E_{\text {relay }}^{(i)}
$$

where, $n_{i}$ is the number of $i^{\text {th }}$ cluster and follows Poisson distribution where $\lambda_{1}=P_{o p t}^{-1}, d_{C H-C H}^{4}(i)$ is distance between $i^{\text {th }}$ cluster head and cluster head receiving from $i^{\text {th }}$ cluster, it is as following, since the area is $\frac{2 a}{\sqrt{P_{o p t} \cdot N}} \times \frac{2 a}{\sqrt{P_{o p t} \cdot N}}$, when cluster head in on the centroid of cluster area.

$$
d_{C H-C H}(i)=\frac{2 a}{\sqrt{P_{o p t} \cdot N}} \Leftrightarrow d_{C H-C H}^{4}(i)=\frac{16 a^{4}}{\left(P_{o p t} \cdot N\right)^{2}}
$$

where, $d_{\text {node-CH }}^{2}(i)$ is the square of distance between sensor nodes and cluster heads and the expected valve of it is as following,

$$
E\left[d_{\text {node-CH }}^{2}(i)\right]=\iint_{A^{\prime}} \sqrt{x^{2}+y^{2}} d x d y=0.765 \frac{a^{2}}{P_{\text {opt }} N}
$$

where, $A^{\prime}$ is $\frac{2 a}{\sqrt{P_{o p t} \cdot N}} \times \frac{2 a}{\sqrt{P_{o p t} \cdot N}}$ area.

Expanding Eq.(6) by using Eq.(7) and (8) is as following, 


$$
\begin{aligned}
E_{\text {net }}= & N P_{o p t}\left[2\left(P_{o p t}^{-1}-1\right) E_{D A}\right)+P_{o p t}^{-1} E_{\text {elec }}+\left(P_{o p t}^{-1}-1\right) E_{\text {cal }} \\
& +\varepsilon_{1} \frac{16 a^{4}}{\left(P_{o p t} N\right)^{2}}+\varepsilon_{2} 0.765 \frac{a^{2}}{P_{o p t} N}\left(P_{o p t}^{-1}-1\right)+\sum_{i} E_{\text {relay }}^{(i)} \\
= & 2 N\left(1-P_{o p t}\right) E_{D A}+N E_{\text {elec }}+N\left(1-P_{o p t}\right) E_{c a l}+\frac{16 \varepsilon_{1} a^{4}}{N P_{o p t}} \\
& +\varepsilon_{2} 0.765 a^{2}\left(P_{o p t}^{-1}-1\right)+\sum_{i} E_{\text {relay }}^{(i)}
\end{aligned}
$$

Since $\sum_{i} E_{\text {relay }}^{(i)}$ is independent of the number of sensor nodes in $i^{\text {th }}$ cluster, total energy consumption in network is a function of $P_{o p t}$, when $N$ is given and independent of the number of sensor nodes in every cluster. From this, researches have been done focusing on calculating $P_{o p t}$, the probability of optimal cluster heads in [23] [25]-[27].

However, unbalance of the number of sensor nodes per cluster affects average energy consumption. Average energy consumption per sensor node in $i^{\text {th }}$ cluster is as following,

$$
\begin{aligned}
\frac{E_{\text {cluster }}^{(i)}}{n_{i}}= & \frac{E_{\text {cluster }}+E_{\text {relay }}^{(i)}}{n_{i}} \\
= & 2\left(1-\frac{1}{n_{i}}\right) E_{D A}+E_{\text {elec }}+\left(1-\frac{1}{n_{i}}\right) E_{\text {cal }}+\varepsilon_{1} \frac{s_{i}^{2}}{n_{i}} \\
& +0.191 \varepsilon_{2}\left(1-\frac{1}{n_{i}}\right) s_{i}+\frac{\sum_{i} E_{\text {relay }}}{n_{i}}
\end{aligned}
$$

That is, the larger $n_{i}$, the higher average energy consumption in cluster.

In dynamic clustering unbalance of average energy consumption of sensor nodes per cluster is mitigated randomly by reclustering continuously, however in static clustering since it rotates cluster heads, but there is no reclustering, unbalance of number of sensor nodes in cluster. Because cluster of more number of sensor nodes are destroyed quickly and it affects the performance of total WSN. When clusters are divided into same size of sensing areas like in FLOG, the number of sensor nodes in every cluster is following Poisson distribution where $\lambda_{1}=P_{o p t}^{-1}$ and both of the expected value and variance of it is $\lambda_{1}$.

That is, FLOG has variance of average energy consumption of sensor nodes as following in terms of unbalance of average energy consumption per sensor node.

Of course, in the proposed method, there is no this variance. The method dividing sensing area into clusters so that average energy consumption per sensor node is equal is NP-hard and is similar to in balance k-clustering algorithm proposed in [29], computation of this method is $O\left(N^{6 k}\right)$.

Cluster dividing algorithm is as follows,

- Divide sensing area into $k\left(=P_{\text {opt }} \cdot N\right)$ clusters evenly. This is same as in FLOG.

- Redivide clusters so that energy consumption per sensor node in every cluster is equal by taking no account of communication between clusters (balanced k-clustering).
- Since energy consumption is proportional to area of cluster, computation on redividing clusters is same as in BAK-C (Balanced K-clustering).

- Readjusts cluster size by taking into account energy consumed in communication between clusters like in [30 33].

$$
\begin{aligned}
& \sigma^{2} \stackrel{\Delta}{=} E\left[\left(\frac{E_{\text {cluster }}^{(i)}}{n_{i}}-E\left[\frac{E_{\text {cluster }}^{(i)}}{n_{i}}\right]\right)^{2}\right] \\
& =E\left[\begin{array}{l}
\left(\begin{array}{l}
2 E_{D A}\left(\frac{1}{n_{i}}-E\left[\frac{1}{n_{i}}\right]\right) \\
+E_{c a l}\left(\frac{1}{n_{i}}-E\left[\frac{1}{n_{i}}\right]\right) \\
+\varepsilon_{1} \frac{16 a^{4}}{p_{o p t}^{2} N^{2}}\left(\frac{1}{n_{i}}-E\left[\frac{1}{n_{i}}\right]\right) \\
+0.765 \varepsilon_{2} \frac{a^{2}}{P_{o p t} N}
\end{array}\right)\left(\frac{1}{n_{i}}-E\left[\frac{1}{n_{i}}\right]\right)^{2}
\end{array}\right] \\
& =\left(\begin{array}{l}
2 E_{D A}+E_{c a l} \\
+\frac{\varepsilon_{1} 16 a^{4}}{P_{o p t}^{2} N^{2}} \\
+0.765 \varepsilon_{2} \frac{a^{2}}{P_{o p t} N}
\end{array}\right)^{2} E\left[\left(\frac{1}{n_{i}}-E\left[\frac{1}{n_{i}}\right]\right)^{2}\right] \\
& =\left(\begin{array}{l}
2 E_{D A} P_{o p t} \\
+E_{c a l} P_{o p t} \\
+\frac{\varepsilon_{1} 16 a^{4}}{P_{o p t} N^{2}} \\
+\frac{0.765 \varepsilon_{2} a^{2}}{N}
\end{array}\right)^{2}
\end{aligned}
$$

Thus, cluster dividing algorithm proposed in this paper is FLOG + BAK-C + EEUC. The proposed algorithm is called EBEC (Energy Balance and Efficient Clustering). In terms of computation necessary to cluster dividing, the total computation of EBEC proposed in this paper is high and computation of individual sensor node is lowest. In other words, although the total computation is high, it makes low demands on sensor nodes and makes exact hardware demands on sinks, since all computation are done in sinks and the sinks report the computation results to sensor nodes. Due to high computation necessary to cluster forming, the time necessary to clustering is long.

However, total time taking to clustering also is short by clustering early and not clustering almost after that, since the proposed method is almost static as semi-dynamic clustering.

Let's consider K-C algorithm of variable weight.

As noted before, the proposed method in this paper is no more than a combination of FLOG, BAK-C and EEUC proposed in previous works. Then the problem how to combine these algorithm is presented. In order to solve this problem, we propose Variable Weight K-C (VW K-C). 
This algorithm is as following,

- Divide sensing area into $k\left(=p_{\text {opt }} \cdot N\right)$ clusters evenly and grant the weight $w_{i}$ expressed by Eq.(10) with $n_{i}\left(\sum_{i} n_{i}=N\right)$ sensor nodes in $i^{\text {th }}(i=\overline{1, k})$ cluster.

- Execute BAK-C algorithm with all $\mathrm{N}$ sensor nodes (with $w_{i}: i=\overline{1, k}$ ) input. That is, if existing BAK-C algorithm is algorithm forming $k$ clusters assuming that all sensor nodes are equal, in this algorithm $\mathrm{N}$ sensor nodes have one weight of $k$ other weights. In actual implementation, we change some codes of $\mathrm{k}$-mean clustering function Kmean $(X, K)$, core of BAK-C algorithm. Grant the weight $w_{i}^{\prime}$ computed by Eq.(10) with $n_{i}$ sensor nodes of k clusters formed newly.

- Compute energy that current clusters consume on relay, add weights $w_{\text {relay }}=\frac{E_{\text {relay }}(i)}{n_{i}}$ to weights of $n_{i}$ sensor nodes in every $i^{\text {th }}$ cluster, $w_{i}^{\prime}$, where $E_{\text {relay }}(i)$ : energy that ith cluster consumes on relay.

- At last, all $n_{i}$ sensor nodes in ith cluster, the result of execution of VW-BAK-C algorithm have weights $w^{\prime \prime}=w^{i}+w_{\text {relay }}$.

- Repeat VW-BAK-C algorithm of second and third step with all $N$ sensor nodes (with $w^{\prime \prime}(j): i=\overline{1, k}$ ) inputs. $w^{\prime \prime}(j)$ is the weights of $n_{i}$ sensor nodes in ith cluster during jth round.

It repeats round until member nodes of all clusters do not change (equilibrium). In practice, stop the round based on one of three conditions, since infinite round occurs or the time to reach equilibrium can be long.

First, repeat round at fixed number.

Second, stop the round, if $\max _{i} \frac{\left|w_{i}^{\prime \prime}(j)-w_{i}(j+1)\right|}{w_{i}^{\prime \prime}(j)} \leq \eta_{1}$

Third, stop the round, if

$$
\frac{1}{N} \sum_{i}\left(\begin{array}{l}
\text { the number of sensor nodes newly } \\
\text { came into } i^{\text {th }} \text { cluster+the number of } \\
\text { sensor nodes went over from } i^{\text {th }} \text { cluster } \\
\text { to other cluster }
\end{array}\right) \leq \eta_{2}
$$

If it stops the round by above conditions, it cannot ensure complete energy balance. This unbalance of energy is mitigated by reclustering.

\subsubsection{Cluster Heads Rotation:}

Since the proposed clustering method in this paper is semidynamic clustering method near static clustering, clusters often do not change. However, cluster heads are rotated. That is, whenever cluster heads are rotated, reclustering are done like in dynamic clustering, clusters remain and it rotates cluster heads of them. In the proposed method in this paper, it rotates cluster heads according to scheduling and whether to rotate or to make a role of cluster head is set so that all sensor nodes of every cluster become cluster heads at most two or three times.
Actual cluster heads rotation is as following,

- After cluster dividing, the sink informs every cluster which cluster given sensor node belongs to and broadcasts schedule about cluster heads rotation to every cluster. Also, the sink informs the number of collections, after which cluster head is changed. Cluster heads receive the gathered data from every sensor nodes according to schedule, process and then send them to the sink.

- If the node making a role of current cluster head is $A$, let us that the node that will make a role of cluster head next is $B$. That is, all sensor nodes are aware that cluster head next $\mathrm{A}$ is $\mathrm{B}$, when the order of cluster head rotation is $A \rightarrow B \rightarrow C$ $\rightarrow D$. However, if $B$ was destroyed or damaged temporally, $A$ did not receive information from target, so it informs to every node in cluster that next cluster head is $C$, considering that $B$ was destroyed.

- If $A$ did not receive data from $C$ either, it informs that next cluster head is $D$. Of course, if $A$ received data from $B$, there is no need to advertise of next cluster head especially. Also, if the number of sensor nodes in its cluster was increased, cluster head reports the fact to the sink and informs all sensor nodes in its cluster.

\subsubsection{Cluster Adjustment-Reclustering:}

The proposed clustering method in this paper is called semidynamic clustering, because it is very near static clustering method and performs reclustering once in a great while in the proposed method. Although the sink constructed clusters so that energy consumption in every cluster is balanced in early stage, average energy consumption of sensor nodes in every cluster is different. Because energy consumption in every cluster is optimal balanced, but it cannot be just the same.

Also, energy consumption of sensor nodes belong to corresponding cluster is high, since the probability of event occurrence in clusters deployed to specific direction is high in detective WSN. In this case the sink can calculate unbalance of energy consumption of sensor nodes previously during cluster forming or based on event data reported and detected during some time. Thus, after some time, it does reclustering in order to remove this unbalance. Of course, this is done in broadcasting total network by calculating previously in the sink.

\subsection{PERFORMANCE EVALUATION OF EBEC}

\subsubsection{Energy Consumption of EBEC:}

Energy is a key concern in all clustering protocols. Of course this results lifetime of sensor nodes and affects the performance of total network. In terms of energy consumption, they mainly have considered energy consumption on transmitting and receiving data after cluster forming in many works.

In this paper, we evaluate of total energy consumption as the sum of energy necessary to cluster forming, transmitting and receiving data after cluster forming and gathering data and calculation.

- Energy necessary to cluster forming.

- Energy necessary to cluster forming can divided into energy necessary to early cluster forming, energy necessary to cluster head rotation and energy necessary to reclustering. 
In the proposed method, energy necessary to first early cluster forming is 0 . Energy necessary to cluster head rotation also is 0 . Only when it is need to change the order of cluster head rotation, energy is consumed on transmitting control packet.

We assume that the length of data packet, QoS packet and control packet are all same. If the probability that one sensor node is damaged per hour is $P_{n f}$ and the length of one frame of cluster head is $l_{C H, P}$, the probability of cluster head broadcast control packet to entire cluster by being changed the order of cluster head rotation is about $P_{n f} \cdot l_{C H, P} \cdot n$. Since $P_{n f}$ is very small, we can ignore energy consumption on transmitting this control packet.

Energy necessary to reclustering is 0 . Because reclustering process is done by the sink and the process is finished for the sink by broadcasting control packet of reclustering to sensor nodes of entire network. The disadvantage of static clustering is additional energy consumption due not to satisfying CNS.

Like in Eq.(1) - Eq.(3), $\varepsilon_{2}(n-1) d_{\text {node-CH}}^{2}$ account for the largest part in energy consumption in cluster. In practice, cluster heads are not on the centroid of cluster in static clustering, so the distances from sensor node to cluster head are different.

Let us consider $d_{\text {node-CH }}^{2}$, in case of most of dynamic clustering where cluster heads are on the centroid of cluster heads and static clustering. That is, we calculate the expected value of the square of distance between $i^{\text {th }}$ node and $i^{\text {th }}$ node and the expected value of the square of distance between $k^{\text {th }}$ node and $j^{\text {th }}$ node, assuming that $j^{\text {th }}$ node is on position $(0.0)$, when there are n nodes in one cluster.

When cluster heads are on the centroid of cluster, that is $j^{\text {th }}$ node is cluster head,

$$
S_{c}=E\left[\sum_{i=1, i \neq j}^{n}\left(x_{i}^{2}+y_{i}^{2}\right)\right]
$$

where, $\left(x_{i}, y_{i}\right)$ is the coordinate of $i^{\text {th }}$ node.

When $k^{\text {th }}$ node is cluster head, $k(k=\overline{1, n}, k \neq j)$

$$
\begin{aligned}
S_{k}= & E\left[\sum_{i \neq k}^{n}\left(x_{i}-x_{k}\right)^{2}+\left(y_{i}-y_{k}\right)^{2}\right] \\
= & E\left[x_{k}^{2}+y_{k}^{2}+\sum_{i \neq k, j}^{n}\left(x_{i}-x_{k}\right)^{2}+\left(y_{i}-y_{k}\right)^{2}\right] \\
= & E\left[x_{k}^{2}+y_{k}^{2}\right]+\sum_{i \neq k, j}^{n} E\left\{\left[\left(x_{i}-x_{k}\right)^{2}+\left(y_{i}-y_{k}\right)^{2}\right]\right\} \\
S_{k}= & \left.E\left[x_{k}^{2}+y_{k}^{2}\right]+2(n-2)\left\{E\left[X^{2}\right]+E\left[Y^{2}\right]\right\}\right\} \\
& =(2 n-3)\left\{E\left[X^{2}\right]+E\left[Y^{2}\right]\right\}
\end{aligned}
$$

Then Eq.(12) is as following,

$$
S_{c}=(n-1)\left\{E\left[X^{2}\right]+E\left[Y^{2}\right]\right\}
$$

That is energy consumption on transmitting from node to cluster head in static clustering is increasing $\frac{2 n-3}{n-1}=2-\frac{1}{n-1}$ times.

\subsubsection{Comparison with Existing Clustering Methods:}

Although the proposed method in this paper is balanced in terms of energy consumption, we cannot consider that it is optimal in terms of energy consumption.

First, let us consider energy consumption on cluster forming in order to compare with energy consumption in representative clustering methods. The lower bound of energy consumption in total cluster forming stage during one cycle is as following, since energy consumption on transmitting and receiving is $(n-1)\left(E_{\text {elec }}+\right.$ $\left.\varepsilon_{2} S_{c}\right),(n-1) E_{\text {elec }}$, respectively.

$$
\begin{aligned}
E_{C F-L B}= & E_{\text {chel }}+n\left(2(n-1)\left(E_{\text {elec }}+\varepsilon_{2} S_{c}\right)+2(n-1) E_{\text {elec }}\right) \\
& =E_{\text {chel }}+2 n(n-1)\left(2 E_{\text {elec }}+\varepsilon_{2} S_{c}\right)
\end{aligned}
$$

where, $E_{\text {chel }}$ is the energy consumption on selecting cluster head during one cycle.

In election of cluster heads based on QoS, we can analyze energy consumption on cluster forming simply compared with random election of cluster heads. Here energy consumption on electing cluster heads during one round is as following, since cluster head select cluster head based on it, after cluster head received QoS frame from (n-1) nodes in its cluster.

$$
\begin{aligned}
E_{\text {chel }}^{\prime}= & n(n-1) K\left(E_{\text {elec }}+\varepsilon_{2} S_{c}\right)+n(n-1) K E_{\text {elec }} \\
& +K(n-1) E_{\text {cal }}+n\left(E_{\text {elec }}+\varepsilon_{c} S_{c}\right)+n(n-1) E_{\text {elec }}
\end{aligned}
$$

where, $K=1 \sim 3,4$ depends on the number of properties such as residual energy, QoS, degrees of nodes, connectivity and so on.

Since minimum value of $K$ is 1 , at least following energy is consumed on electing cluster heads.

$$
\begin{aligned}
& E_{C H e l, L B}^{\prime}=n^{2}\left(E_{\text {elec }}+\varepsilon_{2} S_{c}\right)+2 n(n-1) E_{\text {elec }}+(n-1) E_{\text {cal }} \\
& \underset{E_{\text {cal }}}{\underset{2}{\approx}\left(E_{\text {elec }}\right.}\left(3 n^{2}-2 n\right) E_{\text {elec }}+n^{2} \varepsilon_{2} S_{c}
\end{aligned}
$$

At least energy consumed on cluster forming is as following,

$$
\begin{aligned}
E_{C F-L B}^{\prime}= & E_{C h e l, L B}^{\prime}+n\left(2(n-1)\left(E_{\text {elec }}+\varepsilon_{2} S_{c}\right)+2(n-1) E_{\text {elec }}\right) \\
& =E_{\text {Chel }, L B}^{\prime}+4\left(n^{2}-n\right) E_{\text {elec }}+\left(2 n^{2}-n\right) \varepsilon_{2} S_{c} \\
& =\left(7 n^{2}-6 n\right) E_{\text {elec }}+\left(3 n^{2}-n\right) \varepsilon_{2} S_{c}
\end{aligned}
$$

As known in Eq.(14), Eq.(15), energy necessary to cluster forming is almost 0 , but energy consumed on transmitting data after cluster forming is high in the proposed clustering method in this paper.

Total energy consumption by averaging one data gathering in the proposed method is as following,

$$
\begin{aligned}
E_{E B E C}= & E_{C F, E B E C}+E_{\text {cluster }, E B E C} \\
= & P_{n f} l_{C H, p} \varepsilon_{2} S_{k}+2(n-1) E_{D A}+n E_{\text {elec }} \\
& +\left(l_{C H, P} \approx n\right)+(n-1) E_{\text {cal }}+\varepsilon_{1} d_{C H-C H}^{4} \\
& +\varepsilon_{2}(n-1) S_{k}+E_{\text {relay }}
\end{aligned}
$$

Since the part that $E_{C F, E B E C}$ and $E_{\text {relay }}$ account for total energy consumption is almost 0 , we can approximate as following,

$$
E_{E B C C} \approx E_{\text {cluster }}
$$

Energy consumption by averaging one data gathering in clustering method based on random election of cluster heads is as following, 


$$
\begin{aligned}
E_{\text {rand }}= & \frac{E_{C F-L B}}{K_{1}}+E_{\text {cluster }, \text { rand }} \\
= & \frac{1}{K_{1}}\left[E_{\text {chel }}+\left(4 n^{2}-4 n\right) E_{\text {elec }}+\left(2 n^{2}-2 n\right) \varepsilon_{2} S_{c}\right] \\
& +2(n-1) E_{D A}+n E_{\text {elec }}+(n-1) E_{\text {cal }}+\varepsilon_{1} d_{C H-C H}^{4} \\
& +\varepsilon_{2}(n-1) S_{c}+E_{\text {relay }}
\end{aligned}
$$

where, $E_{\text {cluster, } \text {,rand }}$ is energy consumed on gathering and transmitting data after cluster forming in clustering method based on random election of cluster heads, it is same as $E_{\text {cluster,EBCC }}$ except for $S_{c}$ instead of $S_{k}$. $K_{1}$ is a parameter representing after how times rotates cluster head and is 1 in many protocols such as LEACH and so on, they considered it as 10 at most [24],[34]. The reason why we make $K_{1}$ larger than it is that some nodes are destroyed so quickly due to large unbalance of energy consumption per node, when $K_{1}$ is so large.

Energy consumption by averaging one data gathering in clustering based on cluster head rotation based on QoS is as following,

$$
\begin{aligned}
E_{\text {value }}= & \frac{E_{C F-L B}^{\prime}}{K^{\prime}}+E_{\text {cluster }, \text { value }} \\
= & \frac{1}{K^{\prime}}\left[\left(7 n^{2}-5 n\right) E_{\text {elec }}+\left(3 n^{2}-n\right) \varepsilon_{2} S_{c}\right] \\
& +2(n-1) E_{D A}+n E_{\text {elec }} \\
& +(n-1) E_{\text {cal }}+\varepsilon_{1} d_{C H-C H}^{4}+\varepsilon_{2}(n-1) S_{c}+E_{\text {relay }}
\end{aligned}
$$

where, $E_{\text {cluster, value }}$ is same as $E_{\text {cluster, rand }}$, as energy consumed on gathering and transmitting data after cluster forming in clustering based on cluster head rotation based on QoS and $K^{\prime}$ is parameter representing how times rotates cluster head.

Let us compare $S_{c}$ with $S_{k}$, in order to compare Eq.(19) with Eq.(20) and Eq.(21). As known in Eq.(14) and Eq.(15),

$$
\begin{aligned}
& S_{k}=(2 n-3)\left\{E\left[X^{2}\right]+E\left[Y^{2}\right]\right\} \\
& S_{c}=(n-1)\left\{E\left[X^{2}\right]+E\left[Y^{2}\right]\right\}
\end{aligned}
$$

As seen above, $(x, y)$ is approximately on square area

$$
\left(-\frac{a}{\sqrt{P_{o p t} N}},-\frac{a}{\sqrt{P_{o p t} N}}\right) \sim\left(\frac{a}{\sqrt{P_{o p t} N}}, \frac{a}{\sqrt{P_{o p t} N}}\right)
$$

where, $x, y$ is independent each other and follow uniform distribution.

$$
\begin{gathered}
E\left[X^{2}\right]=E\left[Y^{2}\right]= \\
=\frac{1}{\frac{a}{-\sqrt{P_{o p t} N}}} \alpha^{2} \frac{1}{2 \sqrt{P_{o p t} N}} d \alpha \\
=\left.\frac{a^{3}}{\sqrt{P_{o p t} N}} \alpha^{3}\right|_{-\frac{a}{\sqrt{P_{\text {opp }} N}}} ^{\frac{a}{\sqrt{P_{p p t} N}}} \\
=\frac{a^{3} N}{3 P_{o p t} N}
\end{gathered}
$$

From Eq.(22), Eq.(14) and (15) are as following,

$$
\begin{gathered}
S_{k}=\frac{2(2 n-3) a^{3}}{3 P_{o p t} N} \\
S_{e}=\frac{2(n-1) a^{3}}{3 P_{o p t} N}
\end{gathered}
$$

In practice, Eq.(22) and (23) are lower bound of energy consumption.

The real number of cluster heads is different from $P_{\text {opt }} \cdot N$ in dynamic clustering and energy consumption due to it is not small either. When the real number of cluster heads is not $N P_{\text {opt }}$, additional energy consumption due to it is approximately the square of difference between real ratio of cluster heads and optimal probability of cluster heads [23] [35].

From this, many methods reducing the variance by introducing concept of the variance of the number of cluster heads are presented. In case of the smallest variance, relative standard variance deviation is $7 \sim 10 \%$, that is the ratio of energy loss is $15 \sim 20 \%$ this time. Thus, we have to consider by multiplying Eq.(22) and Eq.(23) and minimum parameter $\gamma=1.15 \sim 2$. At last, the result is as following,

$$
\begin{aligned}
& E_{E B C C}= 2(n-1) E_{D A}+n E_{\text {elec }}+(n-1) E_{\text {cal }} \\
&+\varepsilon_{1} d_{C H-C H}^{4}+\frac{2(2 n-3)(n-1) a^{3} \varepsilon_{2}}{3 P_{\text {opt }} N}+E_{\text {relay }} \\
& E_{\text {rand }}=\frac{\gamma}{K_{1}}\left[E_{C H e l}+\left(4 n^{2}-4 n\right) E_{\text {elec }}+\left(2 n^{2}-2 n\right) \varepsilon_{2} S_{c}\right] \\
&+\gamma(2(n-1)) E_{D A}+n E_{\text {elec }}+(n-1) E_{\text {cal }} \\
&+\varepsilon_{1} d_{C H-C H}^{4}+\varepsilon_{2}(n-1) S_{c}+E_{\text {relay }} \\
&=\frac{\gamma}{K^{\prime}}\left[\left(7 n^{2}-5 n\right) E_{\text {elec }}+\left(3 n^{2}-n\right) \varepsilon_{2} S_{c}\right] \\
& \quad+\gamma\left(2(n-1) E_{D A}+n E_{\text {elec }}+(n-1) E_{\text {cal }}\right. \\
&\left.\quad+\varepsilon_{1} d_{C H-C H}^{4}+\varepsilon_{2}(n-1) S_{c}+E_{\text {relay }}\right)
\end{aligned}
$$

Here, we consider approximately that $d_{C H-C H}=\frac{2 a}{\sqrt{P_{o p t} N}}$, $E_{\text {realy }}=\lambda \varepsilon_{1} d_{C H-C H}^{4}$, where $\lambda$ is a parameter representing how current cluster should relay. The characteristic of the proposed method in this paper is that it concentrates energy consumption and computation to the sink and it minimizes energy consumption and computation on sensor nodes.

\section{SIMULATION RESULTS}

\subsection{ANALYZING CLUSTERING BY EBEC}

The Fig. 1 and Fig. 2 shows example of clustering by EBEC, the clustering method proposed in this paper, and $k$-clustering method, when the number of sensor nodes is 1000 (the number of clusters is 7). The sensor nodes are deployed randomly. As seen in figures, different clusters were formed from k-clustering by implementing EBEC used the weighted Euclid distance. 


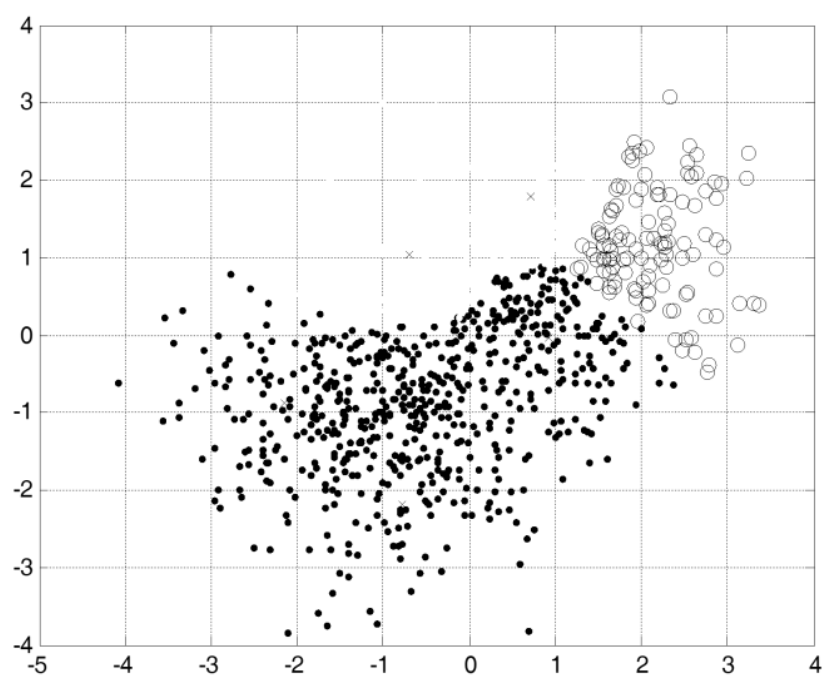

Fig.1. Clustering by k-clustering method, when the number of sensor nodes is 1000

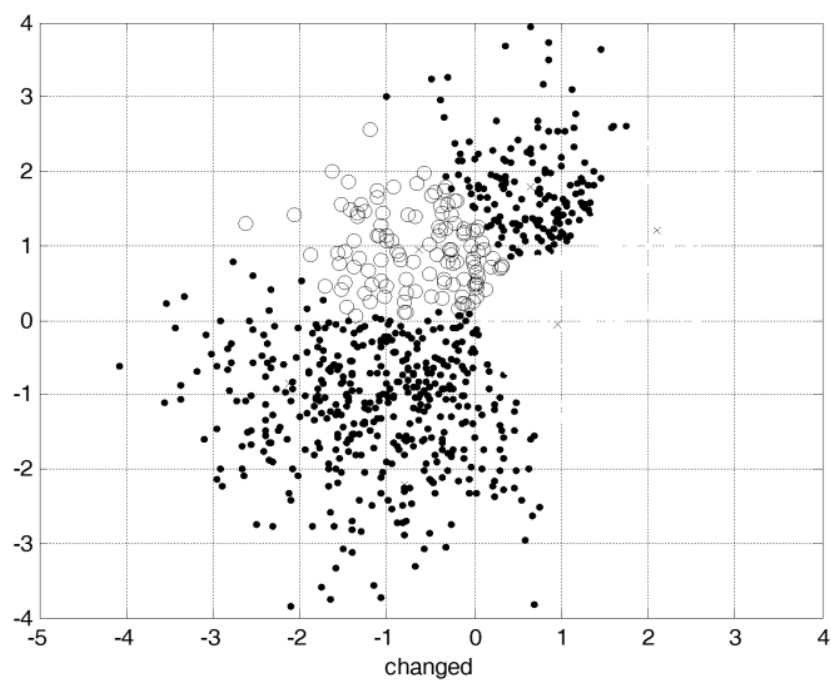

Fig.2. Clustering by the proposed method, when the number of sensor nodes is 1000

\subsection{PERFORMANCE EVALUATION OF EBEC}

We evaluated the performance of EBEC by using MATLAB. We obtained the result by Monte-Carlo simulating 10000 times. The Table. 1 shows simulation parameters.

\subsubsection{Comparison of Energy Consumption of Sensor Nodes:}

The Fig. 3 and Fig.4 shows comparison of energy consumption of sensor nodes of the proposed method and clustering based on random rotation and rotation based on QoS of cluster heads. The number of data gathering is the average number of gathered data occurring during one rotation of cluster heads. In general, the number of data gathering is less than 10 and 20 at most in almost clustering methods.

Table.1. Simulation parameters

\begin{tabular}{|c|c|}
\hline Parameters & Value \\
\hline Network grid & $100 \times 100 \mathrm{~m}$ \\
\hline
\end{tabular}

\begin{tabular}{|c|c|}
\hline Number of nodes & $100 \sim 3000$ \\
\hline Length of Data, $n$ & $4000 \mathrm{bit}(500$ byte $)$ \\
\hline Electronics energy, $E_{\text {elec }}$ & $50 \mathrm{pJ} / \mathrm{bit}$ \\
\hline Data Aggregation energy, $E_{D A}$ & $50 \mathrm{pJ} / \mathrm{bit}$ \\
\hline Transmitter energy, $\varepsilon_{1}$ & $10 \mathrm{pJ} / \mathrm{bit} / \mathrm{m}^{2}$ \\
\hline Amplifier energy, $\varepsilon_{2}$ & $0.0013 \mathrm{pJ} / \mathrm{bit} / \mathrm{m}^{4}$ \\
\hline Initial energy, $E_{\text {init }}$ & $0.5 \mathrm{~J}$ \\
\hline
\end{tabular}

The cluster head should be rotated once in LEACH, 10 11 times in PEGASIS and 11 12 times in ZigBee standard per data gathering, respectively. As shown in Fig. 3 and Fig.4, the proposed method due to these rotation numbers shows more than two times energy performance.

\subsubsection{Unbalance of the Number of Nodes per Cluster:}

In general, the method of the most performance in terms of unbalance of the number of nodes per cluster is k-clustering algorithm. Fig.5. shows unbalance of the number of nodes per cluster.

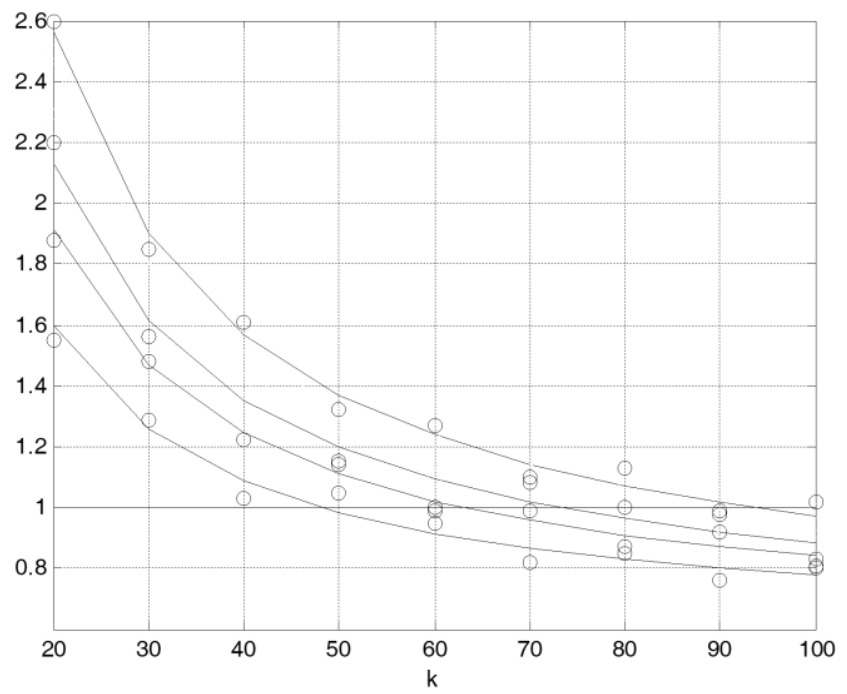

Fig.3. Comparison of average energy consumption per node of the proposed method and clustering method based on random rotation of cluster heads

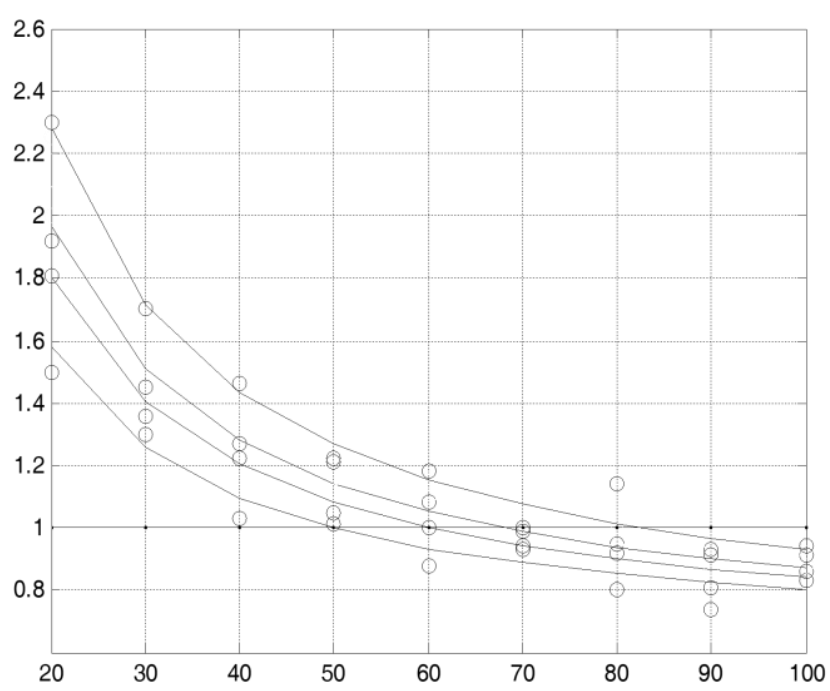


Fig.4. comparison of average energy consumption per node of the proposed method and clustering method based on cluster head rotation based on QoS

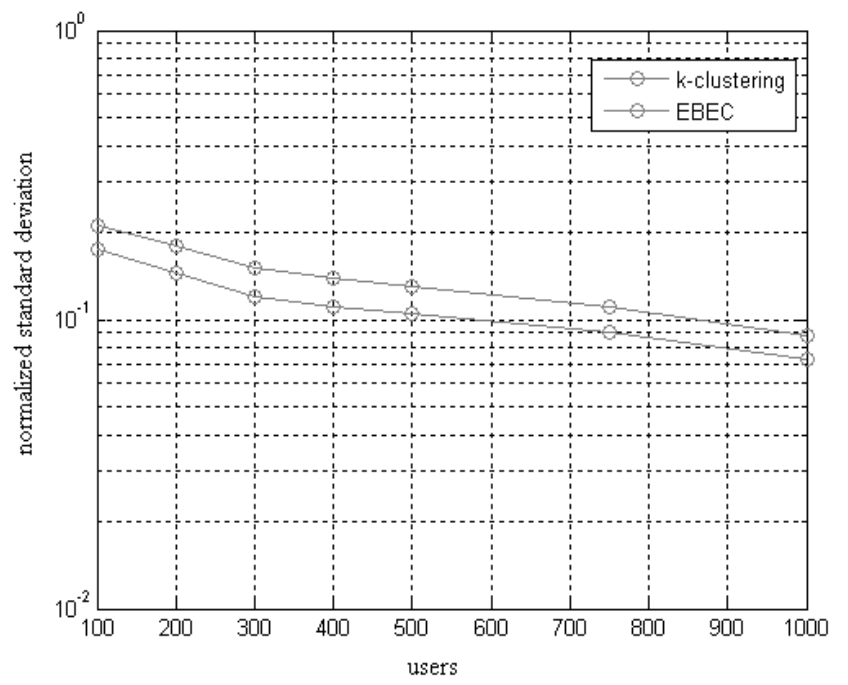

Fig.5. Normalized standard deviation of the number of nodes per cluster for the number of nodes

The larger the number of nodes, the larger the variance of the number of nodes per cluster, in order to consider it quantitatively, we evaluated it as normalized standard deviation not variance.

\section{CONCLUSION}

In this paper, we proposed EBEC clustering method forming clusters based on balance of energy consumption of nodes in WSN. Unlike previous methods, the proposed method is almost static centralized protocol, and its characteristic is that the sinks transmit most of control message and process most of data, which is the major source of energy consumption. As a result, the proposed method has an advantage in that the requirements on the energy and hardware of nodes are not so high. First, we proposed EBEC method that optimizes by considering energy consumption on transmitting and receiving data, energy consumption on reclustering and hot-spot problem that be optimized individually in previous works all together. In order to implement this method, we used VW BAK-C algorithm by introducing the concept of variable weighted Euclid distance to k-clustering algorithm. Second, we classify the previous clustering methods into random method and the method based on QoS according to the characteristic of cluster head rotation and analyzed average of total energy consumption of nodes mathematically. We compared and analyzed the proposed method based on it. Third, we evaluated the performance of the proposed method by comparing the performance of EBEC and other clustering methods through simulation.

\section{REFERENCES}

[1] Jun Zheng and Abbas Jamalipour, "Wireless Sensor Network", John Wiley and Sons, 2007.

[2] Jason Tillett, Raghuveer Rao, Ferat Sahin and T.M. Rao, "Particle Swarm Optimization for the Clustering of Wireless
Sensors", Proceedings of SPIE, Vol. 5, No. 100, pp. 73-83, 2003

[3] Lorenzo A. Rossi and C.C. Jay Kuo, "Semi-Dynamic Approaches to Node Clustering for Sensor Networks", Proceedings of Internet Quality of Service, Vol. 5245, pp. 54-65, 2003.

[4] R. Purtoosi, H. Taheri, A. Mohammadi and F. Foroozan, "A Light-Weight Contention-based Clustering Algorithm for Wireless Ad Hoc Networks", Proceedings of $4^{\text {th }}$ International Conference on Computer and Information Technology, pp. 627-632, 2004.

[5] Indranil Gupta, Denis Riordan and Srinivas Sampalli, "Cluster-Head Election using Fuzzy Logic for Wireless Sensor Networks", Proceedings of $3^{\text {rd }}$ Annual Communication Networks and Services Research Conference, pp. 255-260, 2005.

[6] M. Yang, J. Wang, Z. Gao, Y. Jiang and Y. Kim, "Coordinated Robust Routing by Dual Cluster heads in Layered Wireless Sensor Networks", Proceedings of $8^{\text {th }}$ International Symposium on Parallel Architectures, Algorithms and Networks, pp. 366-372, 2005

[7] Henoc Soude and Jean Mehat, "Energy Efficient Clustering Algorithm for Wireless Sensor Networks", Proceedings of International Conference on Wireless and Mobile Communications, pp. 232-238, 2006.

[8] Yaoyao Yin, Juwei Shi, Yinong Li and Ping Zhang "Cluster Head Selection using Analytical Hierarchy Process for Wireless Sensor Networks", Proceedings of $17^{\text {th }}$ Annual IEEE International Symposium on Personal, Indoor and Mobile Radio Communications, 2006

[9] Xiaobo Chen and Zhisheng Niu, "A Randomly Delayed Clustering Method for Wireless Sensor Networks", Proceedings of IEEE Communications Society, pp. 33533358, 2006.

[10] Weike Chen, Wenfeng Li, Heng Shou and Bing Yuan, "A QoS-based Adaptive Clustering Algorithm for Wireless Sensor Networks", Proceedings of IEEE International Conference on Mechatronics and Automation, pp. 19471952, 2006.

[11] Dawei Xia and Natalija Vlajic, "Near-Optimal Node Clustering in Wireless Sensor Networks for Environment Monitoring", Proceedings of IEEE $21^{\text {st }}$ International Conference on Advanced Information Networking and Applications, pp. 1825-1829, 2006.

[12] L.M.C. Arboleda and N. Nasser, "Comparison of Clustering Algorithms and Protocols for Wireless Sensor Networks", Proceedings of Canadian Conference on Electrical and Computer Engineering, pp. 1787-1792, 2006.

[13] Wenfeng Li, Weike Chen and Xinzhu Ming, "A Localcentralized Adaptive Clustering Algorithm for Wireless Sensor Networks", Proceedings of $15^{\text {th }}$ International Conference on Computer Communications and Networks, pp. 149-154, 2006.

[14] Hang $\mathrm{Su}$ and $\mathrm{Xi}$ Zhang, "Energy-Efficient Clustering System Model and Reconfiguration Schemes for Wireless Sensor Networks", Proceedings of $40^{\text {th }}$ Annual Conference on Information Sciences and Systems, pp. 99-104, 2006.

[15] B. Huang, Fei Hao, Hui Zhu, Yuji Tanabe and B. Takaaki, "Low-Energy Static Clustering Scheme for Wireless Sensor 
Network", Proceedings of $5^{\text {th }}$ International Conference on ITS Telecommunications, pp. 925-930, 2006

[16] Yiping Yang, Chuan Lai and Lin Wang, “An EnergyEfficient clustering Algorithm for Wireless Sensor Networks", Proceedings of $10^{\text {th }}$ International Conference on Control and Automation, pp. 875-879, 2013.

[17] Adel Youssef, Mohamed Younis and Moustafa Youssef, "Distributed Formation of Overlapping Multi-hop Clusters in Wireless Sensor Networks", Proceedings of IEEE Global Telecommunication Conferences, pp. 167-173, 2006.

[18] Na Yao and Laurie Cuthbert, "Reducing Congestion over Hotspot Clusters in WCDMA Networks", Proceedings of IEEE Wireless Communications and Networking Conference, pp. 3731-3735, 2007

[19] Peter Hebden and Adrian R. Pearce, "Distributed Asynchronous Clustering for Self-Organisation of Wireless Sensor Networks", Proceedings of $4^{\text {th }}$ International Conference on Intelligent Sensing and Information Processing, pp. 37-42, 2006.

[20] Yongxuan Lai, Xiaobo Fan, Chen Hong and Ting Xie, "Optimization Framework for Distributed Clustering Scheme in Wireless Sensor Networks", Proceedings of $8^{\text {th }}$ ACIS International Conference on Software Engineering, Artificial Intelligence, Networking, and Parallel/Distributed Computing, pp. 26-31, 2007.

[21] Wei Zhou, Hui-Min Chen and Xue-Fan Zhang, "An Energy Efficient Strong Head Clustering Algorithm for Wireless Sensor Networks", Proceedings of International Conference on Wireless Communications, Networking and Mobile Computing, pp. 2584-2587, 2007.

[22] Jing Deng, Yunghsiang S. Han, Wendi B. Heinzelman and Pramod K. Varshney,"Balanced-Energy Sleep Scheduling Scheme for High Density Cluster-based Sensor Networks", Computer Communications, Vol. 28, No. 14, pp. 1631-1642, 2005.

[23] Xun Su, "A Combinatorial Algorithmic Approach to Energy-Efficient Information Collection in Wireless sensor Networks", ACM Transactions on Sensor Networks, Vol. 3, No. 1, pp. 22-41, 2010.

[24] $\mathrm{Hu}$ Xiangdong, "On-demand local cluster maintenance model and algorithm for Internet aware layer", Journal of Software Chinese, Vol. 26, No. 8, pp. 2020-2040, 2015.

[25] Sankalpa Gamwarige and Chulantha Kulasekere, "Optimization of Cluster Head Rotation in Energy Constrained Wireless Sensor Networks", Proceedings of International Conference on Wireless and Optical Communications, Networking, pp. 342-346, 2007.

[26] Rui Wang, Guozhi Liu and Cuie Zheng, "A Clustering Algorithm based on Virtual Area Partition for
Heterogeneous Wireless Sensor Networks", Proceedings of IEEE International Conference on Mechatronics and Automation, pp. 372-376, 2007.

[27] Changmin Duan and Hong Fan, "A Distributed Energy Balance Clustering Protocol for Heterogeneous Wireless Sensor Networks", Proceedings of International Conference on Wireless and Optical Communications, Networking and Mobile Computing, pp. 2469-2473, 2007.

[28] Jing Deng, Yunghsiang S. Han, Wendi B. Heinzelman and Pramod K. Varshney, "Balanced-Energy Sleep Scheduling Scheme for High Density Cluster-based Sensor Networks", Computer Communications, Vol. 28, No. 14, pp. 1631-1642, 2005.

[29] Jaime Lloret, Miguel Garcia, Diana Bri and Juan R. Diaz, "A Cluster-Based Architecture to Structure the Topology of Parallel Wireless Sensor Networks”, Sensors, Vol. 9, No. 12, pp. 10513-10544, 2009.

[30] Yongxuan Lai, Xiaobo Fan, Hong Chen and Tingt Xie, "Optimization Framework for Distributed Clustering Scheme in Wireless Sensor Networks", Proceedings of $8^{\text {th }}$ International Conference on Software Engineering, Artificial Intelligence, Networking, and Parallel/Distributed Computing, pp. 26-31, 2007.

[31] M. Dhanaraj and C.S.R. Murthy, "On Achieving Maximum Network Lifetime through Optimal Placement of Clusterheads in Wireless Sensor", Proceedings of IEEE International Conference on Communications, pp. 31423147, 2007.

[32] Dali Wei and H. Anthony Chan, "Clustering Algorithm to Balance and to Reduce Power Consumptions for Homogeneous Sensor Networks", Proceedings of International Conference on Wireless and Optical Communications, Networking and Mobile Computing, pp. 2723-2726, 2007.

[33] Soheil Ghiasi, Ankur Srivastava, Xiaojian Yang and Majid Sarrafzadeh, "Optimal Energy Aware Clustering in Sensor Networks", Sensors, Vol. 2, pp. 258-269, 2002.

[34] Y. Wu, Z. Chen, Q. Jing and Y.C. Wang, "LENO: Least Rotation Near-Optimal Cluster Head Rotation Strategy in Wireless Sensor Networks", Proceedings of $21^{\text {st }}$ International Conference on Advanced Networking and Applications, pp. 107-113, 2007

[35] Wei Zhou, Hui-Min Chen and Xue-Fan Zhang, "An Energy Efficient Strong Head Clustering Algorithm for Wireless Sensor Networks", Proceedings of International Conference on Wireless and Optical Communications, Networking and Mobile Computing, pp. 2584-2587, 2007. 